\title{
Atividade Assistida por Equinos em pacientes com transtornos mentais: \\ Implementação de projeto piloto em Unidade Básica de Saúde em Pelotas - RS
}

Equine Assisted Activity in patients with mental disorders: Implementation of a pilot project in a

Basic Health Unit in Pelotas - RS

Actividad Asistida por Equinos en pacientes con trastornos mentales: Implementación de un

proyecto piloto em una Unidad Básica de Salud de Pelotas - RS

Recebido: 14/04/2021 | Revisado: 26/04/2021 | Aceito: 07/05/2021 | Publicado: 21/05/2021

Margarida Aires da Silva

ORCID: https://orcid.org/0000-0002-6304-2465 Universidade Federal de Pelotas, Brasil

E-mail: guidaaires1@gmail.com

Giovana Mancilla Pivato

ORCID: https://orcid.org/0000-0001-9139-3687 Universidade Federal de Pelotas, Brasil

E-mail: gimpivato@gmail.com

Marcos Eduardo Neto

ORCID: https://orcid.org/0000-0002-6030-7130 Universidade Federal de Pelotas, Brasil

E-mail: netomarcoseduardo@gmail.com

Bruna dos Santos Suñe Moraes

ORCID: https://orcid.org/0000-0002-4823-2906 Universidade Federal de Pelotas, Brasil

E-mail: brunasune@hotmail.com

Carlos Eduardo Wayne Nogueira

ORCID: https://orcid.org/0000-0002-8555-7953 Universidade Federal de Pelotas, Brasil

E-mail: cewnogueira@gmail.com

Bruna da Rosa Curcio

ORCID: https://orcid.org/0000-0001-8678-3816 Universidade Federal de Pelotas, Brasil

E-mail: curciobruna@hotmail.com

Lenara Lamas Stelmach

ORCID: https://orcid.org/0000-0003-2625-4204 Universidade Federal de Pelotas, Brasil

E-mail: cewnogueira@gmail.com

Paulo Eduardo Favaretto

ORCID: https://orcid.org/0000-0002-8857-4556 Universidade Federal de Pelotas, Brasil

E-mail: pauloeduardofavaretto@hotmail.com

Carlos Alberto Pupper Bandeira

ORCID: https://orcid.org/0000-0002-4174-1863 Universidade Federal de Pelotas, Brasil E-mail: capbandeira@gmail.com

\begin{abstract}
Resumo
A atividade assistida por animais (AAA) é utilizada como uma interação alternativa as terapias tradicionais, na qual o animal é usado como mediador para estabelecer relações informais com fins recreativos e de entretenimento. Assim, desenvolveu-se este estudo com objetivo analisar a interação entre treze pessoas diagnosticadas com transtornos mentais e a espécie equina, dentro do contexto de atividades assistidas por animais, em uma Unidade Básica de Saúde na cidade de Pelotas-RS. As atividades foram divididas em dois momentos: encontros presenciais utilizando dois equinos selecionados e treinados, e encontros remotos através de plataforma digital durante a pandemia de COVID19. Durante os encontros, presencias e virtuais, foram observados comportamentos e reações dos pacientes e questionamentos sobre suas percepções das atividades. Fizeram parte das avaliações também a equipe de saúde da Unidade e os próprios participantes. Com essa experiência foi possível observar que a intervenção foi positiva para os pacientes, demonstrando a importância dos equinos como mediadores na comunicação e interação social dos participantes com transtornos psiquiátricos. Assim, a atividade assistida por equinos mostrou-se uma potencial alternativa terapêutica em diferentes serviços de saúde mental.
\end{abstract}

Palavras-chave: Atividade assistida por equinos; Transtornos mentais. 


\begin{abstract}
Animal-assisted activity (AAA) is used as an alternative interaction to traditional therapies, in which the animal is used as a mediator to establish informal relationships for recreational and entertainment purposes. Thus, this study was carried out with the aiming analyzing the interaction between thirteen people diagnosed with mental disorders and the equine species, within the context of activities assisted by animals, in a Basic Health Unit in the city of Pelotas-RS. The activities were divided into two moments: face-to-face meetings using two selected and trained horses, and remote meetings through a digital platform during the COVID-19 pandemic. During the meetings, presences and virtual, patients' behaviors and reactions and questions about their perceptions of activities were observed. The health team of the Unit and the patients themselves were also part of the evaluations. With this experience, it was possible to observe that the intervention was positive for patients, demonstrating the importance of horses as mediators in the communication and social interaction of participants with psychiatric disorders. Therefore, equine-assisted activity proved to be a potential therapeutic alternative in different mental health services.
\end{abstract}

Keywords: Equine assisted activity, Psychiatric disorders.

\title{
Resumen
}

Este estudio tuvo como objetivo analizar una interacción entre tres personas diagnosticadas con trastornos mentales y una especie equina, en el contexto de actividades asistidas por animales, en una Unidad Básica de Salud de la ciudad de Pelotas-RS. Las actividades se dividieron en dos momentos: encuentros presenciales con la presencia de dos caballos seleccionados y entrenados, y encuentros remotos a través de una plataforma digital durante la pandemia COVID-19. Durante las reuniones, presenciales y virtuales, se observaron comportamientos y reacciones de los pacientes y preguntas sobre sus percepciones de las actividades. El equipo de salud de la Unidad y los propios participantes también formaron parte de las evaluaciones. Se pudo observar que una intervención positiva para los pacientes, demostró la importancia de los caballos como mediadores en la comunicación e interacción social de los participantes con trastornos psiquiátricos. Con esta experiencia, una actividad asistida por caballos se convierte en una potencial alternativa terapéutica en diferentes servicios de salud mental.

Palabras clave: Actividad asistid por equinos; Trastornos mentales.

\section{Introdução}

O reconhecimento da ligação entre humanos e animais tem estimulado pesquisas aumentando o interesse sobre o impacto de atividades e terapias mediadas por animais com objetivos de reabilitação física, social e bem-estar das pessoas (Faraco et al, 2009). Neste contexto, a abordagem reconhecida como atividade assistida por animais (AAA), uma das modalidades das intervenções que utilizam o animal como parte do processo, está incorporada em contextos de saúde (Ribeiro et al, 2017). Tais intervenções são pensadas como alternativas para algumas das limitações de terapias tradicionais, porque não dependem exclusivamente da linguagem para promover mudanças (Wilson et al, 2016).

Diferente da Terapia Assistida por Animais (TAA) em que a intervenção é direcionada por objetivos e metas específicas para cada indivíduo (Kruger e Serpeel, 2010), a AAA consiste de interações espontâneas de recreação e entretenimento, sem metas por sessão ou registro de evolução (Ribeiro et al, 2017; Scapin et al 2020). Com esse propósito, as atividades assistidas que utilizam a espécie equina como mediadores representam um campo emergente de intervenções assistidas por animais, empregando o uso de cavalos em uma variedade de atividades com humanos (Scopa et al, 2019).

Nesta modalidade, Atividade Assistida por Equinos (AAE), a interação não utiliza técnicas de equitação para reabilitação como na equoterapia (ANDE BRASIL, 2020). Conforme Black 2012, os animais podem agir como facilitadores sociais e potenciais catalisadores para o contato humano-humano, o que influencia a interação social entre pessoas e fatores relacionados como confiança e empatia (Beetz, 2012). Assim, os potenciais benefícios deste contato podem ser especialmente importantes para pessoas com transtornos mentais que normalmente estão associados a sofrimento ou incapacidade, afetando suas atividades sociais e profissionais (American Psychiatric Association, 2014). O presente estudo tem por objetivo verificar a interação entre pessoas com transtornos mentais nas atividades assistidas por equinos, no contexto de uma Unidade Básica de Saúde (UBS). 


\section{Metodologia}

O trabalho foi um estudo de caso qualitativo, realizado no período de setembro de 2019 a dezembro de 2020, com pacientes psiquiátricos da UBS Centro Social Urbano do Areal, na cidade de Pelotas-RS.

\section{Grupo de estudo}

Composto por treze pessoas, adultas, com idades entre 22 e 74 anos, diagnosticadas com transtorno mental, que participam da Oficina de Criação Coletiva da UBS. A estrutura faz parte do programa Estratégia Saúde da Família e desenvolve o projeto Oficinas de Criação Coletiva, que reúne pacientes crônicos portadores de sofrimento psíquico, em reuniões interdisciplinares semanais. A oficina faz parte de um projeto de extensão da Faculdade de Medicina da Universidade Federal de Pelotas (UFPel), do Departamento de Saúde Mental, com apoio do Departamento de Medicina Social, e atende pacientes com diferentes diagnósticos de transtornos mentais como esquizofrenia, transtorno de espectro autista, bipolaridade, Síndrome de Tourette e depressão.

\section{Equipe executora do projeto}

Os executores foram pós-graduandos e graduandos da Medicina Veterinária da UFPel, coordenado pela médica veterinária residente autora deste trabalho e o professor orientador, contando com o apoio da equipe de saúde da UBS, composta pela assistente social e o médico residente da psiquiatra.

\section{Escolha dos animais}

Foram selecionados dois equinos da raça pônei (Figura 1), adultos, de comportamento dócil e sociável. Os equinos foram treinados constantemente, repetindo a rotina que fazem junto aos pacientes. Duas vezes por semana realizavam rotina em área do Hospital de Clínicas Veterinária da UFPel, similar ao que fariam com as pessoas na UBS. Ambos eram avaliados, constantemente, quanto à adequada condição sanitária, e recebiam periodicamente vermífugos e vacinas dentro do protocolo preconizado pela equipe de trabalho.

Figura 1. Equinos da raça Pônei selecionados e treinados para o projeto.

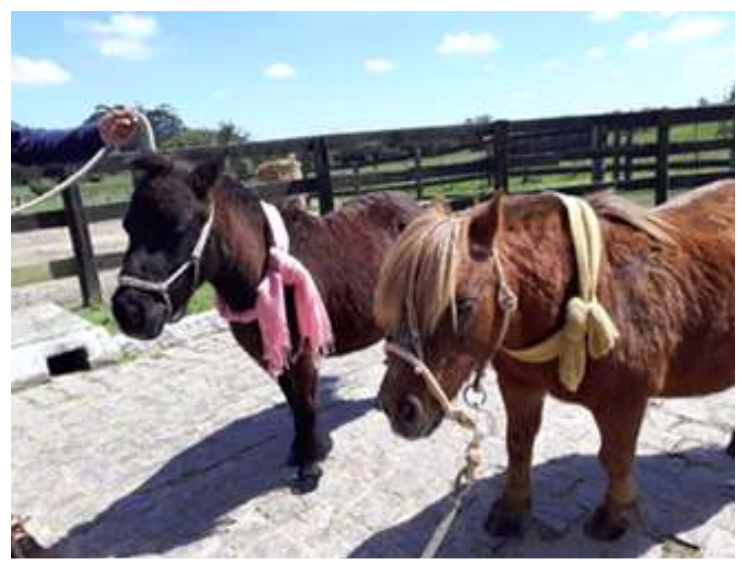

Fonte: Autores. 


\section{Intervenções}

As atividades foram divididas em dois momentos: visitas presenciais e encontros por chamada de vídeo após a imposição do distanciamento social pela pandemia causada pelo COVID-19.

\section{Visitas presenciais}

Foram realizadas cinco visitas com duração de duas horas em uma praça pública próxima à UBS. Em três dos encontros, os pacientes foram estimulados à aproximação, à interação e ao toque nos equinos, com atividades como a escovação dos pelos e carinho nos animais. Dois encontros foram realizados sem a presença dos animais, dentro do ambiente da UBS, um deles com atividades relacionadas ao conhecimento do comportamento e curiosidades sobre a espécie, e outro comemorativo ao encerramento das atividades.

\section{Encontros remotos}

Foi realizado um grupo no aplicativo WhatsApp com os pacientes e a equipe executora e de apoio do estudo, no qual eram divulgados semanalmente conteúdos informativos sobre a espécie equina de forma interativa e lúdica. Além disso, eram realizadas chamadas de vídeo quinzenalmente, num total de 12 encontros, que permitia aos pacientes a interação em tempo real onde eram mostrados os cavalos e realizadas atividades relacionadas. Foram abordados temas como cuidados de saúde com os equinos, manejo, nutrição, curiosidades e peculiaridades da espécie. Além disso, o espaço foi aproveitado para reforçar a necessidade dos cuidados no enfrentamento à pandemia de COVID-19, como a importância do uso de máscara, higiene e distanciamento social.

\section{Avaliações}

Durante as visitas e interações remotas, foram observados os comportamentos e reações dos pacientes durante a interação, e realizados questionamentos informais sobre suas percepções dos encontros. Da mesma forma, a equipe de saúde também contribuiu com suas avaliações sobre a atividade.

\section{Resultados}

Durante os encontros presenciais com os pôneis (Figura 2), a maioria dos pacientes interagiu conforme a proposta, realizando a escovação dos pelos dos animais, fornecendo alimento, passeando e buscando contato físico. 
Figura 2. A, B,C,D,E: Pacientes interagindo com os equinos da raça pônei.

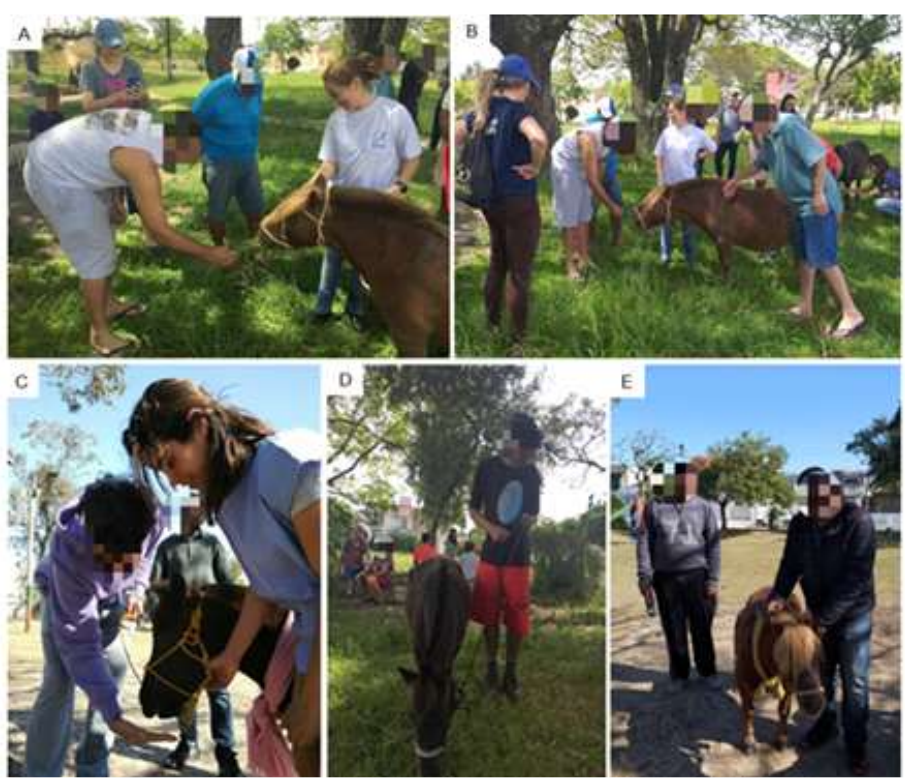

Fonte: Autores.

Foi possível observar que a atividade assistida por equinos proporcionou reações de satisfação demonstradas e expressas verbalmente pelos pacientes na interação com os animais. Os pacientes relataram ser uma experiência nova e positiva a proximidade com equinos de baixa estatura. Além disso, foi relatado também por alguns participantes o contato anterior com a espécie equina, porém não de maneira próxima, com possibilidade de interação, como o contato direto através da escovação dos pelos. Dois participantes diagnosticados com transtorno do espectro autista, alteração caracterizada por déficits na comunicação e interação social, reagiram buscando efetivamente o contato físico com os animais, o que surpreendeu a equipe de saúde da UBS. Já outra paciente autista demonstrou relutância na aproximação durante o primeiro encontro por ter presenciado previamente um acidente envolvendo cavalos. Porém, com o estímulo por parte da equipe envolvida, procurou gradualmente proximidade, embora mantendo a distância que considerava segura.

O comportamento dos dois pôneis durante as intervenções foi essencial para que as pessoas buscassem a aproximação. Os animais mantiveram-se calmos, sem manifestar reações inesperadas frente ao ambiente diferente dos treinamentos, mesmo com a presença e manipulação de pessoas que não estavam acostumados.

No encontro presencial no ambiente da UBS, sem a presença dos pôneis (Figura 3), os pacientes demonstraram interesse pelos materiais ilustrativos que foram apresentados sobre a anatomia, pelagens, e outras curiosidades sobre cavalos.

Figura 3. A e B: pacientes olhando livros sobre cavalos; C: paciente autista desenhando uma "máscara de cavalo".

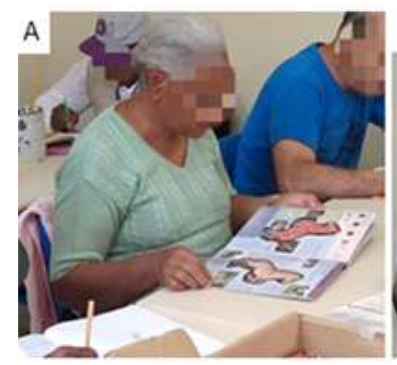

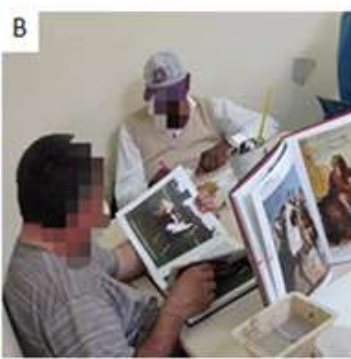

Fonte: Autores.

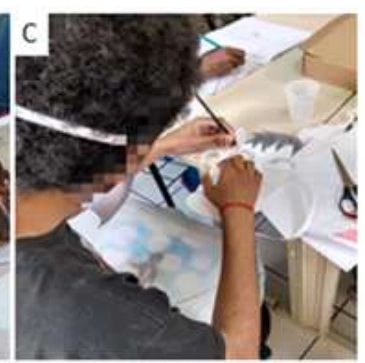
. 
Nos encontros virtuais (Figura 4), foi possível observar um ambiente descontraído durante as intervenções, embora nem todos participassem. Os pacientes realizaram questionamentos sobre as atividades e deram sugestões sobre o que poderia ou gostariam que fosse abordado nas próximas chamadas de vídeo.

Figura 4. Interações por chamada de vídeo durante a pandemia de COVID-19.

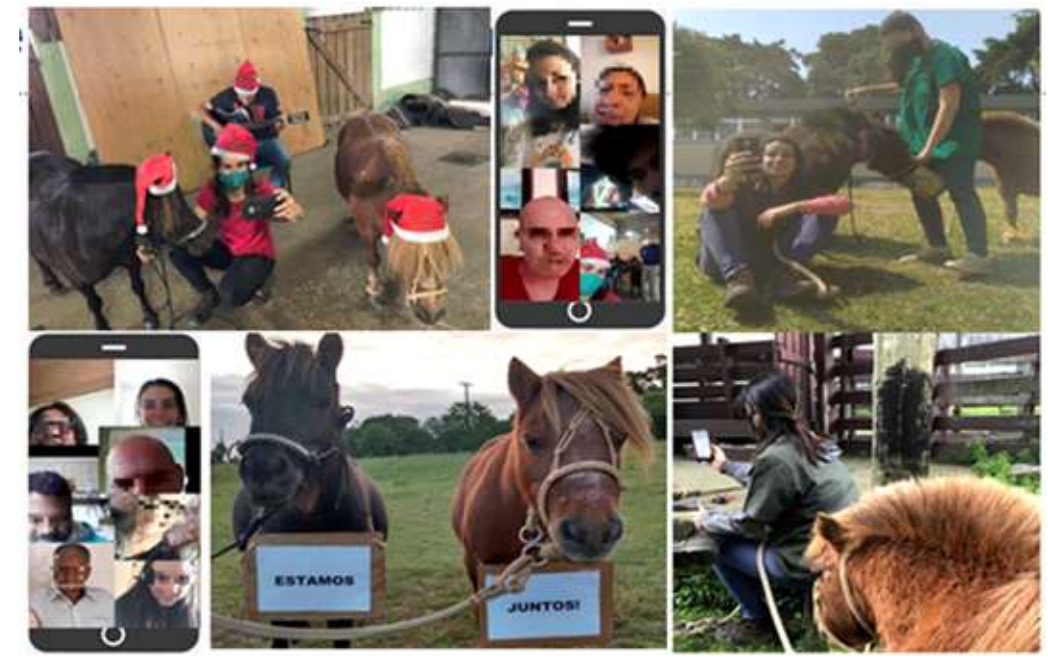

Fonte: Autores.

A avaliação pela equipe de saúde da UBS e dos próprios pacientes foi positiva, conforme os relatos:

"As atividades junto aos cavalos mostraram como o animal é um facilitador de atitudes e troca de comportamento. Isto foi observado na questão do toque no animal de pessoas que têm essa dificuldade com outras pessoas. Isso foi muito claro junto aos autistas. Nesse período de grande dificuldade, da pandemia, foi fundamental para o não isolamento completo do grupo, o que ocasionaria agravamento dos problemas de saúde mental. Não tivemos nenhuma pessoa com quadro agravado ou que necessitasse de internação neste período. A possibilidade de continuar vendo os animais, colegas e técnicos, trouxe alívio de ansiedade e segurança. Sei que é difícil mensurar comportamentos, mas grupos de saúde mental se manifestam de forma direta quando não gostam de uma atividade ou relação com pessoas, o que aqui não ocorreu, houve sempre manifestação de apreço.” (Assistente social da UBS)

"Dentre os transtornos apresentados pelos pacientes, muitos apresentam como sintomas centrais, a dificuldade na interação social e comunicação [...] Quando surgiu a ideia de aplicar ao grupo uma intervenção de AAE, após alguns meses de observação minha desta dinâmica, fiquei curioso para descobrir como seria a reação de pacientes tão distintos. Quando a intervenção ocorreu e os animais foram apresentados ao grupo foi possível notar a diferente reação gerada nos pacientes. [...] O subgrupo de pacientes que apresenta caráter mais introspectivo e dificuldade de socialização curiosamente foi quem iniciou a interação com os animais. Foi possível observar a ocorrência de um certo tipo de facilitação na comunicação com os outros através do animal. A colaboração em alimentar, escovar os pelos e acariciar os equinos aparentemente os fazia interagir de modo mais espontâneo e ativo. De outra forma, pacientes mais extrovertidos apresentaram um certo grau de retração, mas não deixavam de participar da interação social, observando a relação dos demais pacientes com os animais e tecendo comentários sobre. Posteriormente, estes também interagiram diretamente com os "mini cavalos", mas penso que o animal neste caso facilitou-lhes notar mais nitidamente a presença e alteridade destes outros pacientes, balanceando a divisão da atenção que cada um solicita a grupo. Creio que a experiência foi muito instigante, gerando diversas hipóteses observacionais frente à intervenção." (Médico psiquiatra da Unidade)

"Eu acho bom, porque meu colega mesmo que é mais quieto, mais calado, parece que ele participava mais. Uma pena que não podia ser presencial. Acho que os guris participaram mais quando era presencial. Eu acho lindo (cavalo) ao mesmo tempo em que ele mostra liberdade, ele mostra força também. É o que mais gosto (projeto). Eu tenho medo ainda. É muito boa a interação de vocês no grupo. Eu gostei muito. Eu sei que é difícil elaborar um trabalho com um grupo como o nosso, porque são necessidades e idades diferentes e apesar disso, vocês sempre trouxeram algo para todos." (Paciente com transtorno do espetro autista 1)

“Eu acho lindo! Gostei de ambos (presencial e remoto)." (Paciente com transtorno do espetro autista 2) 


\section{Discussão}

A partir deste projeto, pode-se demonstrar que as atividades como a AAE realizada na UBS podem ser incorporadas na saúde e educação para fins terapêuticos, educacionais ou recreativos, pois conforme Kruger \& Serpell 2010, os animais possuem atributos intrínsecos que facilitam a terapia, servindo como mediadores das interações sociais. Desta forma, tal abordagem pode ser um recurso importante para os pacientes com transtornos psiquiátricos dentro do contexto das Oficinas Terapêuticas, que são espaços que tem como objetivo a ressocialização e resgate da cidadania (Azevedo, 2011). Apostando na relevância dos benefícios da interação entre animais e pacientes com transtornos mentais, os trabalhos da Dra. Nise da Silveira, na década de 1950, no hospital psiquiátrico Dom Pedro II, no Rio de Janeiro, foram as primeiras tentativas nacionais do uso dos animais com fins terapêuticos (Garcia \& Botomé, 2008).

Com base nas competências sócio-emocionais dos equinos, que desenvolveram habilidades comunicativas para se relacionar com os humanos, acredita-se que ocorra uma transferência emocional interespecífica durante as intervenções (Scopa et al, 2019). Assim, pesquisas justificaram a ideia de que a espécie equina tem efeito positivo em traços comportamentais, emocionais e habilidades sociais, propondo que o relacionamento que os humanos constroem com os cavalos representa uma maneira de construir confiança, aceitação, e compreensão para com os humanos também (Pelyva et al., 2020). A evolução concomitante dos animais domésticos com os humanos foi o que propiciou o desenvolvimento de relações interespecíficas. Isso sugere que para estabelecer a relação humano-animal de forma eficiente, é importante a troca de comportamentos recíprocos entre os sujeitos envolvidos e a ocorrência de encontros repetidos (Hinde, 1979), tal como foi a sequência de atividades propostas para o grupo.

A dificuldade inicial de aproximação experimentada por uma paciente e posterior progresso para aproximação com o esforço da equipe, corrobora com Hauge et al. (2013), que relata, que além da interação com o animal, o ambiente natural, os arredores e o ambiente social também parecem ser importantes. Esta percepção de que o ambiente é favorável pode influenciar a autoconfiança e realização em uma atividade.

Conforme Payne et al em 2015, o contato corporal constitui um canal de conexão emocional entre os interagentes, a exemplo do ato de escovação dos pelos e toque nos animais oportunizados aos participantes. Assim, cavalos e humanos tornaram-se capazes de criar vínculo (Nummenmaa, 2012). Usando sinais não intencionais, como vocalizações e expressões faciais, pessoas e cavalos foram aperfeiçoando progressivamente as habilidades para comunicar estados afetivos recíprocos (Lagarde, 2005). Equinos expressam seu comportamento e reagem aos sentimentos apresentados por meio da linguagem corporal, dando uma resposta direta no estado emocional da pessoa (Birke et al, 2011). Com isso, a confiança na interação com um cavalo é importante para conseguir que ele coopere (Burgon, 2011).

Manter o vínculo não presencial dos pacientes com os equinos e a equipe executora em pleno distanciamento social imposto pela pandemia de COVID-19, que levou quase todo o planeta a uma crise sanitária e humanitária (Lima, 2020) foi desafiador. Especialmente porque são atividades de interação direta e o contato físico é parte importante da experiência. Com isso, foi fundamental utilizar o recurso da tecnologia na perspectiva de realizar os encontros remotos e manter a comunicação. Frente às situações adversas, como a pandemia, a criatividade torna-se essencial como uma força de saúde mental na perspectiva de atitudes de otimismo e esperança de dias melhores (Zanon, 2020). Embora o contato físico com os animais não tenha sido possível, os pacientes participaram dos encontros virtuais, na forma de questionamentos e curiosidades sobre a espécie.

No Brasil, a inclusão das ações de saúde mental contribuiu para a consolidação da Reforma Psiquiátrica Brasileira (Correia, 2011), havendo a necessidade da reestruturação do modelo de atenção ao portador de transtornos mentais e da humanização do atendimento psiquiátrico no âmbito do SUS, visando à reintegração social do usuário (Brasil, 2000). Assim, dentro do contexto promoção de saúde e uso de terapias alternativas que empregam como mediadores as espécies animais, 
como o cavalo, foi regulamentada em 2019 a Lei $n^{\circ} 15.352$ que dispõe sobre a permissão para a visitação de animais domésticos e de estimação em hospitais privados, públicos, contratados, conveniados e cadastrados no SUS no Estado do Rio Grande do Sul. Desta forma, abrem-se portas para o uso deste recurso em espaços como a UBS.

\section{Conclusão}

A interação positiva proporcionada pela atividade assistida por equinos no contexto estudado sugere uma alternativa terapêutica potencial dentre as já existentes em diferentes serviços de saúde mental. De acordo com os resultados observados nas visitas à Unidade Básica de Saúde, a proposta de estudo poderia ser expandida para outros diferentes grupos e entidades em que as intervenções poderiam ser benéficas. Em abordagens futuras é interessante aplicar o estudo em um grupo com diagnóstico de um específico transtorno mental, homogêneo e observamos ser fundamental a presença de um profissional de saúde mediador entre os pacientes e a equipe executora.

\section{Referências}

American Psychiatric Association (2014) Manual diagnóstico e estatístico de transtornos mentais/DMS-5.

Associação Nacional de Equoterapia ANDE-BRASIL. Equoterapia. http://equoterapia.org.br/articles/index/articles_list/138/81/0.

Azevedo, D. M., \& Miranda, F. A. N. (2011) Oficinas terapêuticas como instrumento de reabilitação psicossocial: percepção de familiares. Escola Anna Nery, $15(2), 339-345$

Birke, L., Hockenhull, J., Creighton, E., Pinno, L., Mee, J., \& Mills, D. (2011) Horses' responses to variation in human approach. Applied Animal Behaviour Science, 134, 56-63.

Black, K. (2012) The relationship between companion animals and loneliness among rural adolescents. Journal of Pediatric Nursing, $27,103-112$.

Brasil. (2000) Portaria $n^{o}$ 106, de 11 de fevereiro de 2000. http://portalarquivos.saude.gov.br/images/pdf/2015/marco/10/PORTARIA-106-11-FEVEREIRO2000.pdf

Burgon, H. L. (2011) 'Queen of the world': Experiences of 'at-risk' young people participating inequine-assisted learning/therapy. Journal of Social Work Practice, 25, 165-183.

Correia, V. R., Barros, S., \& Colvero, L. de A. (2011). Saúde mental na atenção básica: prática da equipe de saúde da família. Revista da Escola de Enfermagem da USP, 45(6), 1501-1506.

Faraco, C. B., Pizzinato, A., Csordas, M.C., Moreira, M.C., Zavaschi, M. L. S., Santos, T., Oliveira V. L. S., Boschetti F. L., \& Menti, L. M. (2009) Terapia mediada por animais e saúde mental: um programa no Centro de Atenção Psicossocial da Infância e Adolescência em Porto Alegre-TAA Parte III. Saúde Coletiva, 6(34), 231-236.

Garcia, M. P., \& Botomé, S. P. (2008) Da domesticação à terapia: o uso de animais para fins terapêuticos. Interação Em Psicologia, $12(1), 165-167$.

Hauge, H., Kvalem, I. L., Berget, B., Enders-Slegers, M.-J., \& Braastad, B. O. (2013) Equine-assisted activities and the impact on perceived social support, self-esteem and self-efficacy among adolescents - an intervention study . International Journal of Adolescenceand Youth, 19(1), 1-21.

Hinde, R.A. (1979). Towards Understanding Relationships.

Kruger K. A., \& Serpeel, J. A. (2010) Animal-assisted interventions in mental health: Definitions and theoretical foundations. Handbook on Animal-Assisted Therapy: Theoretical Foundations and Guidelines for Practice, 33-48.

Lagarde, J., Peham, C., Licka, T., \& Kelso, J. S. (2005) Coordination dynamics of the horse-rider system. Journal of Motor Behavior, 37, 418-424.

Lima, N. T., Buss, P. M., \& Sousa, R. P. (2020) A pandemia de COVID-19: uma crise sanitária e humanitária. Cadernos de Saúde Pública, 36 (7).

Nummenmaa, L., Glerean, E., Viinikainen, M., Jaaskelainen, I. P., Hari, R., \& Sams, M. (2012) Emotions promote social interaction by synchronizing brain activity across individuals. Proceedings of the National Academy of Sciences, 109(24), 9599-9604.

Payne, E., Boot, M., Starling, M., Henshall, C., Mclean, A., Bennett, P., \& Mcgreevy, P. (2015) Evidence of horsemanship and dogmanship and their application in veterinary contexts. The Veterinary Journal, 204, 247-254.

Pelyva, I. Z., Kresák, R., Szovák, E., \& Tóth, Á. L. (2020) How Equine-Assisted Activities Affect the Prosocial Behavior of Adolescents. International Journal of Environmental Research and Public Health, 17(8), 2967.

Ribeiro, V., Nobre, M. O., Capella, S., \& Vieira, A. C. G. (2017) Interação lúdica na atividade assistida por cães em pediatria. Enfermagem em Foco (Brasília), 8(1): 7-11. 
Research, Society and Development, v. 10, n. 6, e7410615058, 2021

(CC BY 4.0) | ISSN 2525-3409 | DOI: http://dx.doi.org/10.33448/rsd-v10i6.15058

Rio Grande do Sul (2019) Lei 1532. https://leisestaduais.com.br/rs/lei-ordinaria-n-15352-2019-rio-grande-do-sul-dispoe-sobre-a-permissao-para-a-visitacaode-animais-domesticos e\%E2\%80\%A6)

Sapin, C. da F., Lima, C. M., Costa, J. C., Costa, M., Almeida, D. M., Mechereffe, B. M. \& Nobre, M. de O. (2020) Atividade assistida por animais: uma estratégia para pacientes com transtornos mentais. Research, Society and Development. 9(11).

Scopa, C., Contalbrigo, L., Greco, A., Lanatà, A., Scilingo, E. P., \& Baragli, P. (2019) Emotional Transfer in Human-Horse Interaction: New Perspectives on Equine Assisted Interventions. Animals, 9(12), 1030.

Serpell, J. (2002) In the Company of Animals: A Study of Human-Animal Relationships. Livro.

Wilson, K., Buultjens, M., Monfries, M., \& Karimi, L. (2016). Equine-Assisted Psychotherapy for adolescents experiencing depression and/or anxiety: A therapist's perspective. Clinical Child Psychology and Psychiatry, 22(1), 16-33.

Zanon, C. (2020) COVID-19: implicações e aplicações da Psicologia Positiva em tempos de pandemia. Estudos de Psicologia (Campinas) 15(2), 339-345. 